\title{
Archives
}

\section{Mémoire présenté dans le cadre de la consultation publique sur le renouvellement de la politique culturelle du Québec}

\section{Marc Pelchat}

Volume 47, numéro 2, 2018

\section{MÉMOIRES SUR LE RENOUVELLEMENT DE LA POLITIQUE \\ CULTURELLE DU QUÉBEC (2016)}

URI : https://id.erudit.org/iderudit/1045170ar

DOI : https://doi.org/10.7202/1045170ar

Aller au sommaire du numéro

Éditeur(s)

Association des archivistes du Québec (AAQ)

ISSN

0044-9423 (imprimé)

2369-9256 (numérique)

Découvrir la revue

Citer cet article

Pelchat, M. (2018). Mémoire présenté dans le cadre de la consultation publique sur le renouvellement de la politique culturelle du Québec. Archives, 47(2), 77-89. https://doi.org/10.7202/1045170ar
Résumé de l'article

S'appuyant sur une brève rétrospective, le mémoire de l'Église catholique de Québec insiste d'abord, dans la première de ses neuf recommandations, sur la nécessité de l'intégration explicite du patrimoine religieux à la politique culturelle. Il aborde ensuite le rôle de l'état et des collectivités dans la protection, la conservation et la mise en valeur de ce patrimoine archivistique, mobilier et immobilier aussi bien qu'immatériel, insistant sur la faiblesse des moyens et des ressources qu'on y consacre. Après un bref chapitre sur le développement des programmes de sensibilisation et de formation au patrimoine religieux, le mémoire revient sur l'épineuse question des mesures financières à développer ou à créer, tout en reconnaissant les apports financiers de source gouvernementale déjà affectés à la conservation du patrimoine immobilier paroissial. Le mémoire se conclue en rappelant que le patrimoine religieux est un précieux héritage pour le futur, que les églises sont des lieux porteurs d'histoire aussi bien que symboles identitaires et que l'Église catholique de Québec ne peut conserver et valoriser ce riche patrimoine seule, sans aide. 


\section{MÉMOIRE}

\section{Mémoire présenté dans le cadre de la consultation publique sur le renouvellement de la politique culturelle du Québec}

\section{MARC PELCHAT}

Vicaire général, Archidiocèse de Québec

Président du Comité diocésain du patrimoine religieux et d'art sacré

\section{INTRODUCTION - CONTEXTE DE LA DÉMARCHE DE CONSULTATION}

En 1992, le gouvernement du Québec adoptait une première politique culturelle. En 2000, le rapport de M. Roland Arpin Notre patrimoine,

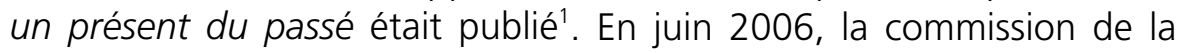
culture de l'Assemblée nationale présentait le rapport Croire au patrimoine religieux du Québec. En 2011, le gouvernement du Québec adoptait la Loi sur le patrimoine culturel du Québec (RLRQ, chapitre P-9.002).

Voilà un rappel de quelques évènements et décisions, parmi tant d'autres, qui ont permis au cours des 25 dernières années de développer un regard collectif sur notre culture et de prendre conscience de l'enjeu du patrimoine religieux au sein de notre société. À l'occasion du renouvellement de la politique culturelle, l'Église catholique de Québec, 
par son Comité diocésain du patrimoine religieux et d'art sacré, souhaite de nouveau contribuer à la réflexion en déposant ce mémoire centré sur la question du patrimoine religieux, notamment immobilier, qui constitue une part incontournable du patrimoine culturel québécois.

\section{RECONNAISSANCE DU PATRIMOINE RELIGIEUX DE L'ÉGLISE CATHOLIQUE DE QUÉBEC}

Au sujet du patrimoine religieux, le Groupe-conseil sur la Politique du patrimoine culturel du Québec, sous la présidence de M. Roland Arpin, écrivait: "Ce patrimoine est le plus universel, le plus diversifié, le plus riche et le plus répandu au Québec. Sa valeur repose sur cinq grands critères: I'intérêt architectural, l'intérêt historique et artistique, I'intérêt urbanistique, et le rôle social et culturel». Quarante et une églises paroissiales dans le diocèse de Québec sont protégées en vertu des dispositions de la Loi sur le patrimoine culturel du Québec (RLRQ, chapitre P-9.002) à titre de biens immobiliers classés, encore situés dans des sites patrimoniaux ou cités par les municipalités.

De même, le tome III des Chemins de la Mémoire publié en 1999, recense les œuvres et objets d'art de vingt églises ${ }^{2}$ de notre diocèse qui ont été classés biens culturels entre 1961 et 1992, soit un tiers des biens mobiliers québécois visés par cette Loi. Ce sont des sculptures (maîtresautels, retables, baldaquins, bancs d'œuvre, mobilier, statues, etc.), peintures, pièces d'orfèvrerie, orgues, ornements sacerdotaux produits par des artistes et artisans et hérités de toutes les époques de notre histoire. Cette contribution de l'Archidiocèse de Québec au patrimoine québécois s'explique aisément par sa longue présence ici, laquelle est indissociable de la vie sociale et culturelle des Québécois.

En effet, l'Église catholique de Québec demeure la plus ancienne institution de notre société tirant ses origines de la présence des missionnaires récollets et jésuites de même que des œuvres des Augustines et des Ursulines établies ici respectivement en 1615, 1625 et 1639. Vicaire apostolique en 1658, François de Laval devint en 1674 le premier évêque d'un diocèse maintenu sans discontinuité jusqu'à aujourd'hui. L'Archidiocèse de Québec a donné naissance, par divisions territoriales successives, à plus de cent cinquante diocèses répartis partout au Canada et aux États-Unis, dont dix-huit au Québec même. Ses 233 églises 
paroissiales actuelles, réparties dans 188 paroisses, témoignent de sa forte présence sur son territoire ${ }^{3}$, sans compter les communautés religieuses qui y tiennent de nombreuses résidences, couvents et monastères. C'est donc à bon droit que l'Église catholique de Québec estime être porteuse d'un patrimoine culturel inestimable par ses biens, certes, mais surtout par sa mission spirituelle et missionnaire menée auprès de toutes les générations depuis les fondements de la Nouvelle-France. L'Église catholique réaffirme entre autres que la dimension spirituelle et religieuse des personnes et des communautés humaines, l'égalité des personnes, le respect de la religion, la prédominance de la langue française, la protection de l'environnement et la recherche du développement durable sont toutes des valeurs qui doivent être rappelées et reflétées dans nos politiques, visions et orientations.

\section{Recommandation 1:}

Que la politique culturelle du Québec intègre explicitement le patrimoine religieux puisqu'il reflète les valeurs spirituelles et humaines de notre société et en raison de la place prédominante qu'il occupe dans notre patrimoine sous toutes ses acceptions.

\section{RÔLE DE L'ÉTAT À L'ÉGARD DU PATRIMOINE RELIGIEUX}

L'élargissement du champ d'application de la Loi sur le patrimoine culturel du Québec (RLRQ, chapitre P-9.002), le réaménagement des statuts de protection, le développement des approches de protection, en engageant davantage le monde municipal et les citoyens et citoyennes, sont des pas importants qui ont été franchis au cours des 25 dernières années.

\subsection{Patrimoine archivistique}

L'Église catholique de Québec rappelle que les archives documentent la présence matérielle de l'Église à travers ses propriétés foncières, ses bâtiments, ses œuvres d'art et objets de culte. Elles rappellent les rites et pratiques de son œuvre pastorale: œuvres missionnaires, éducatives et sociales, œuvres d'adoration et de prière, œuvre d'accueil et de partage. Les archives expriment le message évangélique et demeurent le signe de l'expression de la foi dans notre société ${ }^{4}$. La conservation et la mise en valeur des archives religieuses présentent de multiples défis, notamment 
pour les archives des instituts de vie consacrée alors que plusieurs maisons, couvents et monastères doivent fermer. Les archives paroissiales vont aussi présenter des défis croissants en regard de leur conservation suite aux regroupements juridiques.

\subsection{Patrimoine immobilier}

L'Église catholique de Québec reconnaît l'importance du palier municipal en matière de reconnaissance et de protection du patrimoine religieux bâti. Toutefois, les municipalités ont besoin d'être accompagnées et de disposer de ressources appropriées à ce mandat.

Le réseau municipal québécois est étroitement associé au réseau paroissial, notamment en milieu rural et en dehors des grands centres urbains où territoires et populations coïncident. En outre, c'est aux municipalités qu'incombe la responsabilité d'établir les schémas d'aménagement (MRC) et les plans d'urbanisme (niveau municipal), lesquels devraient impérativement pourvoir à la protection des bâtiments recelant archives et œuvres d'art ainsi qu'à celle des sites et des paysages d'intérêt, car ces derniers traduisent tout autant I'histoire que les valeurs qui sont nôtres. Partant du principe que le patrimoine découle d'une appropriation collective, il devrait appartenir à chaque collectivité locale de définir son patrimoine paysager et de veiller à sa protection et à sa transmission.

\section{Recommandation 2:}

Que la politique culturelle reconnaisse le rôle primordial des collectivités locales dans la définition, la protection et la transmission du patrimoine religieux, et ce, en partenariat et dans le respect des institutions relevant des diverses traditions religieuses, dont l'Église catholique.

\subsection{Patrimoine immatériel}

Le patrimoine immatériel est une dimension reconnue comme importante et essentielle dans la Loi sur le patrimoine culturel du Québec (RLRQ, chapitre P-9.002). Les documents de consultation sur le renouvellement de la politique n'abordent pas clairement ce type de patrimoine. Dans le rapport $\operatorname{Arpin}^{5}$, le patrimoine immatériel est associé 
au patrimoine vivant, fait de savoirs et de savoir-faire traditionnels, qui se fixe sur un support tangible comme les collections des Archives de folklore de I'Université Laval où l'on consulte des transcriptions, photographies et enregistrements sonores ou visuels de contes, chansons, coutumes qui ont eu cours chez nous. L'approche de $\mathrm{M}^{\mathrm{me}}$ Diane Audy, ethnologue, définit le patrimoine immatériel comme suit: "ensemble de traditions, de paroles, de gestes, de pratiques, de rituels et de coutumes ${ }^{6}$. » À cet égard, l'Église a développé un ensemble fort riche de rites et pratiques autant pour le besoin du culte que pour l'accomplissement de ses tâches missionnaires, éducatives et sociales. Combien d'aspects de la vie paroissiale, tout autant que de la vie des communautés, ne sauraient faire sens et resteraient incompris des générations futures sans une attention particulière à ces porteurs de mémoire que sont les membres du clergé, des communautés religieuses et des laïcs associés à la vie religieuse, tels les sacristains et les agents de pastorale par exemple? Force est de constater encore aujourd'hui, chez les jeunes notamment, une ignorance totale des pratiques séculaires, des symboles et des objets sacrés.

Comme le signale à plusieurs reprises $\mathrm{M}^{\mathrm{me}}$ Audy dans l'article cité sur le patrimoine immatériel, c'est par l'enquête orale, les sessions de sensibilisation et les expositions commentées que l'on peut transmettre le véritable sens de nos rites, pratiques et objets de culte, le véritable sens aussi, pourrions-nous ajouter, des représentations artistiques qui ont embelli nos églises, couvents et monastères. Pensons également aux films des abbés Albert Tessier et Maurice Proulx qui ont su capter les gestes traditionnels au moment même où notre société passait à l'ère industrielle. Tous les produits, ne l'oublions pas, résultant de ces enquêtes orales, fixés sur un support analogique ou numérique, et tous ces films constituent aussi des archives qu'il faut s'efforcer de conserver au risque de pertes irréparables. L'Église catholique de Québec est préoccupée par les difficultés d'identification, voire de protection, du patrimoine immatériel lié aux manifestations, aux lieux et même aux personnes porteuses de mémoire.

\section{Recommandation 3}

Que la politique culturelle énonce des orientations et des objectifs à l'égard du patrimoine immatériel, notamment celui de l'Église catholique qui est éminemment présent à travers ses œuvres pastorales, paroissiales, missionnaires, éducatives et sociales. 


\subsection{Collaboration et partenariat}

La Loi sur le patrimoine culturel du Québec (RLRQ, chapitre P-9.002) a confié au monde municipal l'adoption des mesures comme la citation d'un monument historique et la constitution d'un site du patrimoine. D'autres notions sont véhiculées dans d'autres lois québécoises, telles que la protection du patrimoine local ou régional ; l'intérêt d'ordre historique, culturel, esthétique ou écologique (dans la Loi sur l'aménagement et l'urbanisme (RLRQ, chapitre A-19.1)); les études d'impact sur les éléments du patrimoine culturel, archéologique et historique (dans la Loi sur la qualité de l'environnement (RLRQ, chapitre Q-2)); la protection des paysages humanisés (dans la Loi sur la conservation du patrimoine naturel (RLRQ, chapitre (-61.01)); et, finalement, la protection du patrimoine culturel constitué de biens, de lieux, de paysages, de traditions et de savoirs (dans la Loi sur le développement durable (RLRQ, chapitre D-8.1.1)). Conséquemment plusieurs ministères, organismes gouvernementaux, MRC et municipalités sont non seulement concernés, mais appelés à collaborer.

\section{Recommandation 4}

Que la politique culturelle énonce un devoir de conduite et une responsabilité d'harmonisation et de concertation par le gouvernement québécois et ses ministères, particulièrement le ministère de la Culture et des Communications et le ministère des Affaires municipales et de I'Occupation du territoire, en matière de patrimoine religieux, et ce, en partenariat essentiel avec l'Église catholique, pour viser ensemble la protection de ce patrimoine important dans la culture et l'histoire du Québec.

\section{Recommandation 5}

Que soit constituée une instance nationale et permanente de concertation et de dialogue entre l'Église catholique, les autres traditions religieuses, le gouvernement du Québec et ses ministères, dont le ministère de la Culture et des Communications et le ministère des Affaires municipales et de l'Occupation du territoire, pour établir des orientations gouvernementales et définir des priorités d'action en matière de patrimoine religieux. 


\section{INVENTAIRES, ÉTAT DES LIEUX ET PLAN DE CONSERVATION}

En 2005, les premières recommandations de la Commission de la culture ${ }^{7}$ insistaient sur la confection des inventaires, voire d'un inventaire des inventaires. Une meilleure connaissance de nos biens culturels, quels qu'ils soient, demeure la clé de leur protection.

Plusieurs types d'inventaires existent, tout autant que de disciplines reliées au patrimoine. II y a des inventaires archivistiques, constitués par divers organismes: mentionnons le centre des archives de l'Archidiocèse de Québec (relocalisé en 2014 au Pavillon Camille-Roy du Séminaire de Québec), les archives de Bibliothèque et Archives nationales du Québec (BAnQ), les centres régionaux d'archives.

Du côté du patrimoine religieux bâti, le Conseil du patrimoine religieux du Québec diffuse depuis le milieu des années 2000 un inventaire des lieux de culte comportant des données relativement sommaires.

Pour les biens historiques et artistiques, divers inventaires existent. Ces inventaires ont été réalisés au cours des années par des universités, les diocèses ou encore par des organismes comme, par exemple, la Société des musées du Québec.

Enfin, mentionnons l'important Répertoire culturel du Québec qui est une source incontournable de renseignements et de connaissance de notre patrimoine.

L'Église catholique de Québec encourage ses fabriques paroissiales à compléter un «carnet de santé » de leurs bâtiments, mais les coûts associés à cette opération retardent son application. Elle les invite aussi à se doter d'un plan directeur immobilier afin d'évaluer leur besoin réel, établi en relation avec leur projet pastoral, en vue de formuler une appréciation de l'avenir de leurs églises. De même, l'archidiocèse comme les paroisses disposent $d^{\prime}$ 'un guide de gestion de leurs archives ${ }^{8}$ tant pour les documents administratifs courants que pour les documents historiques, mais, encore là, la modestie des ressources disponibles en fait un instrument inégalement appliqué. Notre Église s'est aussi montrée proactive en convenant des ententes de partenariat avec la Ville de Québec, la MRC de Portneuf et plusieurs autres instances publiques et privées pour assurer la sauvegarde d'églises menacées. Finalement, elle 
procède à un relevé complet des meubles, objets de culte et œuvres d'art conservés dans nos églises.

Toutefois, la cueillette d'informations sur l'état actuel des biens susceptibles de protection et la qualité de leur conservation est loin d'être complétée. La mise à jour des inventaires existants est très variable et peu priorisée.

Pour les églises paroissiales, diverses méthodes de hiérarchisation des lieux de culte en raison de leur intérêt patrimonial ont été développées, mais elles ne prennent pas en compte leur état physique. Là encore, un important travail reste à réaliser.

\section{Recommandation 6}

Que la politique mette l'accent sur une stratégie priorisant la poursuite des inventaires ainsi que la réalisation d'un état des lieux du patrimoine religieux et que le gouvernement et le ministère de la Culture et des Communications accordent les ressources financières nécessaires à la réalisation de ces interventions.

\section{CONSULTATION ET PRISE DE DÉCISION AU SUJET DU PATRIMOINE RELIGIEUX}

Malgré l'actuelle Loi sur le patrimoine culturel du Québec (RLRQ, chapitre P-9.002), l'Église de Québec constate les faibles moyens et ressources accordés par le gouvernement pour la protection et la mise en valeur du patrimoine religieux ou encore pour le maintien d'usage des églises paroissiales, particulièrement en milieu rural.

Lors du processus d'attribution d'aide ou de mise en place de partenariat entre les paroisses et les municipalités, la compréhension des critères qui guident les fonctionnaires des différentes institutions ou des directions du ministère de la Culture et des Communications ou du ministère des Affaires municipales et de l'Occupation du territoire n'est pas toujours claire et leur interprétation est variable. Les politiques ne s'inscrivent pas toujours dans une vision partagée des valeurs communes à notre société et à l'Église.

L'Église catholique de Québec souhaite que tous les efforts soient entrepris pour assurer une meilleure cohésion et une bonne collaboration de tous les intervenants gouvernementaux et municipaux. 


\section{Recommandation 7}

Que la politique précise clairement le rôle de l'Église catholique et des autres traditions religieuses, tout autant que celui des intervenants gouvernementaux et municipaux dans le processus de consultation et de décision en matière de patrimoine religieux.

\section{RENFORCEMENT DE L'ENGAGEMENT EN MATIÈRE DE SAUVEGARDE DU PATRIMOINE}

L'engagement individuel ou collectif envers le patrimoine doit être valorisé dans notre société. On a évoqué l'urgence de constituer des inventaires et autres moyens de connaissance. II importe en effet que ces instruments soient le plus accessibles possible pour les citoyens et les chercheurs, mais ce, toutefois, dans le respect des dispositions légales en vigueur en matière de protection des renseignements personnels, des droits d'auteur et de la propriété privée.

Si l'engagement en matière de protection du patrimoine est affaire de citoyens, cette préoccupation est aussi grandement partagée par les institutions que sont les universités, les institutions muséales et culturelles ainsi que leurs constituantes, les Églises et autres institutions religieuses. À cet égard, nous devons souligner les apports du milieu universitaire et des différentes traditions religieuses à la connaissance et à la promotion du patrimoine religieux. Combien de colloques, conférences, rencontres et publications au Québec, sur le patrimoine religieux au cours des dernières années ont été initiés par des professeurs, des chaires spécialisées ou par le Conseil du patrimoine religieux du Québec.

\section{Recommandation 8}

Que la politique réaffirme l'importance que les milieux de formation académique développent des programmes de sensibilisation et de formation au patrimoine religieux à l'intention des étudiants en général, mais aussi des responsables et bénévoles engagés dans les paroisses catholiques ou dans des organisations civiles qui ont à gérer l'important patrimoine mobilier et immobilier de l'Église catholique, qu'il soit encore en usage à des fins religieuses ou qu'il soit désacralisé. 


\section{FINANCEMENT}

Les mesures financières à développer ou à créer pour assurer la conservation des biens culturels du Québec, dont les biens religieux, sont incontournables.

II est vrai que les ressources financières sont limitées, comme nous le rappelle constamment le gouvernement. Mais l'État a une responsabilité de mobilisation et de direction dans la recherche de solutions qui impliquent entre autres les collectivités, les entreprises et les personnes en mesure de contribuer par le mécénat. II a aussi le devoir de rendre accessibles les programmes d'aide existants qui, malheureusement, excluent de façon systématique les paroisses et les diocèses. Trop souvent sont exclus les biens religieux sous prétexte qu'ils sont déjà couverts par le seul et unique programme de "Soutien à la restauration du patrimoine religieux » du Conseil du patrimoine religieux du Québec. Encore là, ce programme ne finance que les travaux de restauration des églises cotées A, B ou C, soit uniquement une partie de celles qui ont été construites avant 1945 (sauf une quarantaine d'églises construites entre 1946 et 1977 ayant une cote $A$ dite incontournable) de sorte que les églises cotées $D$, $E$ et $F$ de même que celles construites après 1945 ne sont admissibles à aucun programme d'aide.

L'Église catholique de Québec reconnaît les apports financiers de source gouvernementale affectés à la conservation du patrimoine immobilier paroissial. Cependant, cet apport est nettement insuffisant pour couvrir les besoins d'un parc patrimonial constitué de 233 églises. De plus, nous devons déplorer le peu de ressources financières qui sont consacrées à la conservation et la mise en valeur du patrimoine archivistique.

\section{Recommandation 9}

Que la politique énonce une stratégie pour développer des nouvelles mesures de financements adéquates et accessibles. Cette stratégie doit identifier spécifiquement le patrimoine religieux. Les mesures d'aide doivent prendre en compte les aspects non couverts par le programme existant du CPRQ et couvrir notamment les frais d'exploitation et les coûts d'énergie liés à la conservation du patrimoine immobilier en priorisant les églises classées, la réalisation des inventaires et états des lieux, la conservation et la diffusion 
du patrimoine archivistique et l'instauration de programmes de sensibilisation et de formation au patrimoine.

\section{CONCLUSION}

Le 2 novembre 2005, les représentants de l'Église catholique de Québec rencontraient les membres de la Commission parlementaire de la culture dans le cadre de la consultation sur le patrimoine religieux du Québec. Les principes alors exposés demeurent encore aujourd'hui les mêmes. Ainsi :

1. Le patrimoine religieux constitue un héritage riche et précieux pour les générations actuelles et futures. II révèle que la dimension spirituelle et religieuse de l'être humain est une composante essentielle à la vie des personnes et des collectivités. Ce patrimoine participe et agit activement dans les cœurs et les esprits. II ouvre le chemin du respect, de la dignité, de l'ouverture à l'autre. II contribue à construire la foi, la charité et l'espérance;

2. Les églises, peu importe leur âge ou leurs qualités au plan architectural ou artistique, méritent d'être considérées comme des lieux porteurs d'une histoire et comme symboles identitaires qui témoignent de la culture, de la vie communautaire et spirituelle d'un milieu. Tout en reconnaissant que l'ensemble des biens religieux ne pourra être conservé, les choix devront favoriser le développement du tissu social des communautés chrétiennes et des collectivités;

3. Les paroissiens, les membres de leur fabrique ou les collectivités en général sont les premiers acteurs dans la recherche de solutions et, en conséquence, tous les efforts doivent être pris pour les soutenir et les reconnaître;

4. Le partenariat est le mot clef dans la recherche de solution. II doit se vivre dans le respect des convictions et droits de chaque partenaire ${ }^{9}$.

C'est donc à la lumière de ces principes que nous avons voulu participer à la présente consultation sur le renouvellement de la politique culturelle.

Le patrimoine religieux occupe sans contredit un espace majeur non seulement par la quantité et la qualité des œuvres architecturales et artistiques qu'il déploie dans tout le Québec, mais aussi dans la perception généralement admise du patrimoine chez nos concitoyens. 
De tout temps, l'Église catholique de Québec a su se doter de biens mobiliers et immobiliers nécessaires à sa mission pastorale, missionnaire, éducative et sociale avec le concours de la population et des institutions publiques. Ces biens, elle les a entretenus et enrichis au fil des années de telle sorte qu'ils constituent aujourd'hui la part la plus visible et la plus répandue de notre patrimoine culturel comme l'ont souvent répété plusieurs auteurs, historiens et spécialistes de l'art sous toutes ses formes.

Toutefois, comme institution, I'Église catholique de Québec reconnaît qu'elle ne peut plus assumer seule la charge de conserver et de valoriser le patrimoine religieux qui est sien et dont elle est la gardienne au nom de sa mission et pour le bien de la collectivité québécoise.

C'est pourquoi elle souhaite que s'instituent une coopération et un partenariat exemplaires entre tous les intervenants - citoyens, institutions, sociétés et associations, municipalités, État -, tous soucieux de la protection de ce patrimoine.

Marc Pelchat

\section{NOTES}

1. GROUPE-CONSEIL SUR LA POLITIQUE DU PATRIMOINE CULTUREL DU QUÉBEC. (2000). Rapport Arpin «Notre patrimoine, un présent du passé». Québec, Québec: Ministère de la Culture et des Communications, p. 191.

2. Chapelle de I'Hôtel-Dieu de Québec, église Saint-Nicolas, église Saint-Louis (Isle-auxCoudres), église Saint-Joseph (Deschambault), église Saint-Elzéar (Beauce), église Saint-Pierre (Île d'Orléans), église Saint François de Sales (Neuville), église SaintFrançois de Sales (Île d'Orléans), église Saint-Henri (Lévis), église Notre-Dame de Lorette (Wendake), chapelle Bon-Pasteur (Québec), église Saint-Michel (Sillery), église Saint-Augustin de Desmaures, église Saint-Jean-Baptiste (Québec), église Sainte-Marie (Beauce), église Saint-Georges (Beauce), église Sainte-Famille (Cap-Santé), église Saint-François (Beauceville), église Saint-Charles-Borromée (Grondines), chapelle des Ursulines (Québec).

3. L'Archidiocèse recouvre quatre régions administratives (Capitale nationale, Centredu-Québec, Chaudière-Appalaches et Estrie).

4. HÉON, G. (2005). Les défis de la conservation des archives religieuses. dans L. Turgeon (dir.), Le patrimoine religieux du Québec: entre le cultuel et le culturel. Québec, Québec: Les Presses de l'Université Laval, p. 310. 
5. GROUPE-CONSEIL SUR LA POLITIQUE DU PATRIMOINE CULTUREL DU QUÉBEC. (2000). Rapport Arpin «Notre patrimoine, un présent du passé». Québec, Québec: Ministère de la Culture et des Communications, 34e recommandation, p. 200.

6. AUDY, D. (2005). Les défis de la conservation des archives religieuses. dans L. Turgeon (dir.), Le patrimoine religieux du Québec: entre le cultuel et le culturel. Québec, Québec: Les Presses de l'Université Laval, p. 225.

7. COMMISSION PERMANENTE DE LA CULTURE. (2006). Croire au patrimoine religieux du Québec. Québec, Québec: Secrétariat des commissions de l'Assemblée nationale du Québec, recommandations 4 et 5.

8. Le Guide de gestion des archives comprend un plan de classification et un calendrier de conservation, lequel prévoit la durée de conservation et la disposition finale des documents.

9. COMITÉ DIOCÉSAIN DU PATRIMOINE RELIGIEUX DE L'ÉGLISE CATHOLIQUE DE QUÉBEC. (2005). Mémoire du Comité diocésain du patrimoine religieux de l'Église catholique de Québec à l'intention de la Commission de la culture de l'Assemblée nationale dans le cadre de sa consultation sur le patrimoine religieux du Québec. Québec, Québec. 